\title{
The universal growth rate behavior and regime transition in adherent cell colonies
}

\author{
F.H.S. Costa ${ }^{\text {a,* }}$, M. Campos ${ }^{\mathrm{b}}$, M.A.A. da Silva ${ }^{\mathrm{a}, \mathrm{c}}$ \\ ${ }^{a}$ Departamento de Física, FFCLRP; Universidade de São Paulo, 14040-901; Ribeirão Preto, São Paulo, Brazil \\ b Departamento de Química e Ciências Ambientais, IBILCE, Universidade Estadual Paulista Júlio de Mesquita Filho, 15054-000 São José do Rio Preto, \\ São Paulo, Brazil \\ ${ }^{\mathrm{C}}$ Departamento de Física e Química, FCFRP; Universidade de São Paulo, 14040-903; Ribeirão Preto, São Paulo, Brazil
}

\section{H I G H L I G H T S}

- We present an analysis of the growth rate of adhrent cell colonies.

- We show that five cell lineages share the same temporal behavior of the growth rate.

- Kinetic parameters in our model are estimated using the experimental data.

\section{A R T I C L E I N F O}

\section{Article history:}

Received 28 February 2015

Received in revised form

17 September 2015

Accepted 25 September 2015

Available online 22 October 2015

Keywords:

Growth rate

Cancer

Mathematical model

\begin{abstract}
A B S T R A C T
In this work, we used five cell lineages, cultivated in vitro, to show they follow a common functional form to the growth rate: a sigmoidal curve, suggesting that competition and cooperation (usual mechanisms for systems with this behavior) might be present. Both theoretical and experimental investigations, on the causes of this behavior, are challenging for the research field; since the sigmoidal form to the growth rate seems to absorb important properties of such systems, e.g., cell deformation and statistical interactions. We shed some light on this subject by showing how cell spreading affects the radius behavior of the growing colonies. Doing numerical time derivatives of the experimental data, we obtained the growth rates. Using reduced variables for the time and rates, we obtained the collapse of all colonies growth rates onto one curve with sigmoidal shape. This suggests a universal-type behavior, with regime transition related to a morphological transition of adherent cell colonies.
\end{abstract}

(c) 2015 Elsevier Ltd. All rights reserved.

\section{Introduction}

Mathematical modeling of tumor growth has been an issue over decades, this has broken down the barriers between experimental, theoretical and computational sciences, and helped us to establish new connections between biology, physics and mathematics (Byrne, 2010). Especially in the last fifteen years, the search for universality classes has made this field of research highly fertile (Brú et al., 1998, 2003; Huergo et al., 2010, 2011, 2012). However, an alternative approach, paying attention to the radius growth rate of the colonies, could be used in the search for essential mechanisms that would rule the dynamics and the morphology of the aggregates of cancerous cells. In fact, the rate at which the number of cells increases or decreases has always been a central point used to evaluate the mechanisms involved

\footnotetext{
* Corresponding author.

E-mail addresses: fhscosta@usp.br (F.H.S. Costa), maasilva@fcfrp.br (M.A.A. da Silva).
}

in the growth of a population (Freyer and Sutherland, 1986), or the effectiveness of a specific treatment (Leith et al., 1993). Sometimes, the approach to the rate is done without taking into account the details that lead to the formation of aggregates, e.g., neglecting the cell to cell interactions (Dawson and Hillen, 2006).

Over the last 20 years, there has been an increasing interest in the study of interactions between cells and in the formation of monolayer aggregates. These studies are done, for example, to refine the margins of irradiation (Unkelbach et al., 2014); to verify possible tumor infiltrations in the healthy tissue (Poplawski et al., 2009); to quantify instabilities in the contour (Amar et al., 2011; Chatelain et al., 2011); changes in the cell cycle (Kim et al., 2014); or to identify known patterns (Brú et al., 1998, 2003; Huergo et al., 2011, 2012). In particular, in the study of monolayer cluster formation, when one does a modeling that includes the effects of proliferation and diffusion explicitly, a wide range of parameters can be chosen to fit the experimental data (Maini et al. 2004a,b). Some experimental work has been done to achieve clear rules for 
its determinations and reduce the multiplicity of choices (Simpson et al., 2013; Treolar et al., 2014).

An alternative approach to deal with aggregates is to propose phenomenological equations using effective growth rates (Drasdo and Hoëhme, 2005; Radszuweit et al., 2009). In the work of Costa et al. (2013) one proposes that the radius increasing rate has a sigmoidal shape. This could be a successful way to describe the time evolution of tumor colonies, since the experimental data and the theoretical modeling agreed quantitatively. Also, one has the advantage of describing this effective sigmoidal rate with continuous functions instead of step-like functions. Moreover, this type of rate behavior suggests the existence of a regime transition that we believe to be related with a morphological transition of cell colonies (Puliafito et al., 2012). Our goal in this work is to extend this approach to several cell lines, and show that the sigmoidal rate behavior is common, being an important intrinsic feature of this type of growth. Also, we discuss the possible mechanisms that may generate this type of behavior ubiquitously present in biochemical reactions, i.e., cooperative and competitive mechanisms.

\section{Model and methods}

\subsection{In vitro experiments and image processing}

We cultivated HeLa (human cervical carcinoma), HCT-115 (human colorectal adenocarcinoma), ${ }_{1}^{1}$ B16-F10 (mouse melanoma) ${ }^{2}$ and $\mathrm{NIH}-$ HN-13 (human head and neck squamous carcinoma) cells (passage 220). Also, we used data from HT-29 (human colon carcinoma) cell lineage (Brú et al., 2003). Cells were plated at low density (25-50 cells/ $\mathrm{ml}$ ) in corning culture flasks with a $25 \mathrm{~cm}^{2}$ growth area. For HeLa and $\mathrm{NIH}-\mathrm{HN}-13$ cell lines we used $4 \mathrm{ml}$ of DMEM medium containing 10\% fetal bovine serum (FBS) and 1\% penicillin and streptomycin; for HCT115 and B16-F10 cell lines we used $4 \mathrm{ml}$ of RPMI-1640 medium with the same amount of supplements mentioned above. Cells were maintained in an atmosphere of $5 \% \mathrm{CO}_{2}$ and $37{ }^{\circ} \mathrm{C}$. During the experiment the whole culture medium was carefully replaced every 3 days.

After $24 \mathrm{~h}$ of culture, several colonies with at least two cells were selected and imaged regularly with magnifications of $10 \times$ and $20 \times$ using two inverted microscopes: one with phase-contrast (Olympus IX-71, to image HeLa, HCT-115 and NIH-HN 13 cells) at the Centro de Pesquisas em Oncologia Molecular (CEPOM), Hospital de Câncer de Barretos-SP-Brazil, and the other equipped with DIC (Nikon Ti-Eclipse, to image B16-F10 cells) at the Laboratório de Química Analítica, Grupo de Física Biológica, Departamento de Física e Química, FCFRP/USP-SP-Brazil. Photographs were scanned with a resolution of $1 \mu \mathrm{m} /$ pixel $(10 \times)$ and $0.5 \mu \mathrm{m} /$ pixel $(20 \times)$ (IX-71), and, $0.64 \mu \mathrm{m} /$ pixel $(10 \times)$ and $0.32 \mu \mathrm{m} /$ pixel $(20 \times)$ (Ti-Eclipse). The colonies' profiles were hand-traced with the aid of Image ${ }^{3}$ and CellSens 2.0 software and the radius measurements of these profiles were done with inhouse computer software. When necessary, the entire image of each colony resulted from the composition of partial images.

With our in-house software tool, we calculated the mean radius for the $10 \times$-profiles using the relation (Costa et al., 2013) $r(t)=\sqrt{2} d_{0} r_{g}$; where $d_{0}$ is the resolution in $\mu \mathrm{m} /$ pixel and $r_{g}=$ $\sqrt{\left(1 / n_{p}\right) \sum_{p=1}^{n_{p}}\left[\vec{r}_{p}-\vec{r}_{c m}(t)\right]^{2}}$ is the radius of gyration, obtained with the pixel's positions $\vec{r}_{p}$, the number of pixels $n_{p}$, and the center of mass $\vec{r}_{c m}(t)=\left(1 / n_{p}\right) \sum_{p=1}^{n_{p}} \vec{r}_{p}$. Each growing colony was followed until reaching the border of a neighboring colony, sometimes taking approximately $330 \mathrm{~h}$ to have contact. For

\footnotetext{
${ }^{1}$ ATCC, Rockville, MD.

2 BCRJ, Rio de Janeiro, Brazil.

3 http://rsbweb.nih.gov/ij/
}

magnifications of $20 \times$ we manually counted the number of cells in the samples.

\subsection{Mathematical model}

As presented in previous work (Costa et al., 2013), we assume an effective rate described by the sigmoidal function:

$\omega(t)=\frac{d}{d t} r(t)=\alpha-\frac{\beta}{1+\exp \left[\gamma\left(t-t_{c}\right)\right]}$.

Initially, the growth is roughly linear with a constant rate $\omega_{0} \equiv \omega(t) \approx \alpha-\beta$. After a critical time $t_{c}$ (note that $\omega\left(t_{c}\right)=\alpha-\beta / 2$ ), the curve changes its behavior going to a constant rate $\alpha$, where $\gamma$ is a parameter that determines how fast the rate evolves from $\omega_{0}$ to $\alpha$. Now, we can define the reduced variables for the rate and time, respectively, as follows:

$\omega_{c}=\frac{[(\omega / \alpha)-1]}{\Gamma}$,

and

$\tau=\gamma\left(t-t_{c}\right)$,

with $\Gamma=\beta / \alpha$. After some algebraic manipulations with these variables, by substituting in Eq. (1), we obtain

$\omega_{c}=-\frac{1}{1+\exp (\tau)}$

Fig. 1 illustrates the biological meaning of the four parameters in Eq. (1), by giving a pictorial view of a growing colony in five different instants.

Thus, despite the different parameters $\alpha, \beta, \gamma$ and $t_{c}$, intrinsic for each cell lineage, the reduced rate and time allow us to collapse the data onto a single sigmoidal-like growth rate curve. Therefore, we could fit the experimental data for the mean radius of the colonies with the function

$r(t)=r_{0}+\frac{\beta}{\gamma} \ln \left\{\frac{\exp \left[-\gamma\left(t-t_{c}\right)\right]+1}{\exp \left(\gamma t_{c}\right)+1}\right\}+\alpha t$,

which is the solution of Eq. (1) with the condition $r(0)=r_{0} \equiv 5 \mu \mathrm{m}$ (Drasdo and Hoëhme, 2005). For the curve fitting, ${ }^{4}$ we did a shift in the data of the mean radius, for different colonies, of each cell line, following a similar approach of Brú et al. (1998) and Puliafito et al. (2012). There, the authors did shifts in the growth curves to coincide with the initial radius at $t=0$ (Brú et al.), and at the beginning of the morphological transition (Puliafito et al.). Here, we do shifts and fittings to build the growth curves, using the following scheme:

(i) make a data fitting for each individual colony with the function given by Eq. (5);

(ii) choose the best curve from the fittings of step (i) - that one with the smallest errors in the parameters - to use as a guide-curve;

(iii) minimize the sum of the residuals, between the guide-curve and the experimental data, of each individual colony, by doing temporal shifts and updating the colonies data;

(iv) put all final data together (including those used to build the guide curve), and make the fitting to obtain the values of the parameters $\alpha, \beta, \gamma$ and $t_{c}$.

\section{Results}

Fig. 2 shows the growth curves and the parameters of the sigmoidal rate (see Eq. (1)). For B16-F10 cells we found $\alpha=6.2 \pm 0.4 \mu \mathrm{m} / \mathrm{h}$. Also, we averaged the parameters obtained for each individual colony (recipe in the step (i) of the shift-fit scheme),

\footnotetext{
${ }^{4}$ To see the data the reader is referred to the Online Supplementary Material.
} 


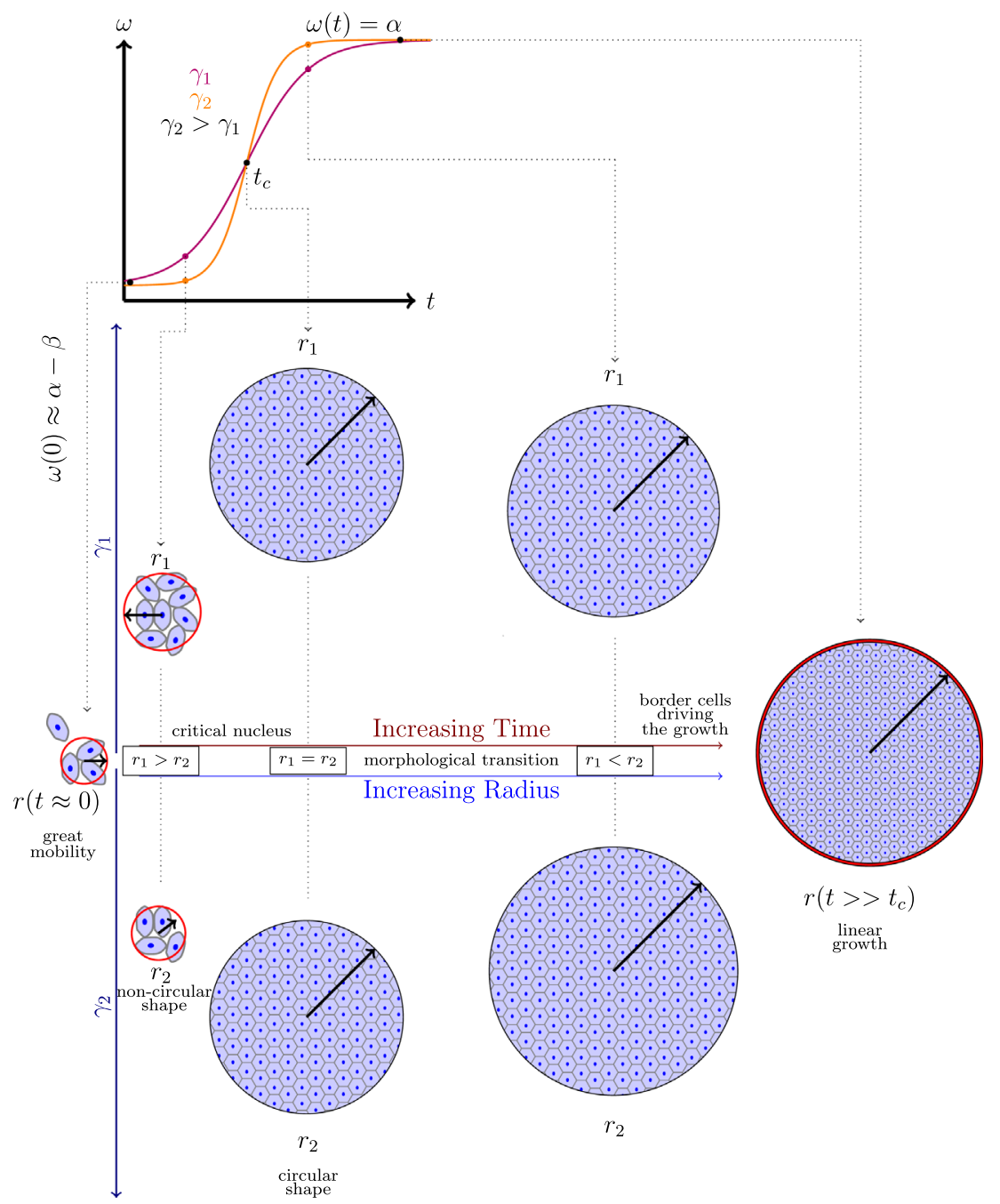

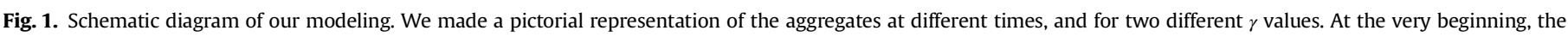

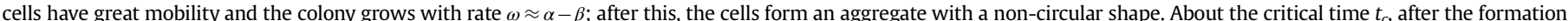

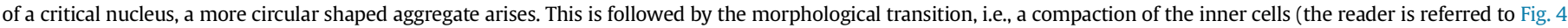

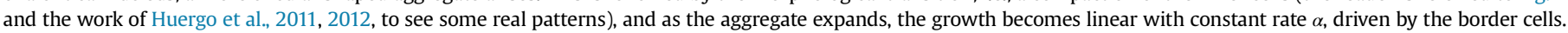

getting $\langle\alpha\rangle=5.7 \pm 0.3 \mu \mathrm{m} / \mathrm{h}$; for the HeLa lineage we obtain $\alpha=$ $2.5 \pm 0.1 \mu \mathrm{m} / \mathrm{h}$ and $\langle\alpha\rangle=2.5 \pm 0.2 \mu \mathrm{m} / \mathrm{h}$, and, for HCT-115 cells, $\alpha$ $=2.4 \pm 0.3 \mu \mathrm{m} / \mathrm{h}$ and $\langle\alpha\rangle=2.6 \pm 0.7 \mu \mathrm{m} / \mathrm{h}$. We present especially the parameter $\alpha$ because we will compare it with the literature in the discussion section; see other parameters in Fig. 2, Table 1, and in the Supplementary Data.

Fig. 3 shows the behavior of the mean radius $R$ as a function of the total number of tumor cells $N_{T}$. We could find that the function $R=R_{0}+a \sqrt{N_{T}}$ fits the data well (Huergo et al., 2011). The parameter $R_{0}$ would be presumably the colony radius obtained by extrapolation to a situation where there would be no cells. However, this has no meaning for the system under consideration, since it requires at least one tumor cell to start the growth process, and so defining a colony. We can replace the radius of the first cell by the relation $R_{\text {eff }}=R_{0}+a$, and thus obtain $R=R_{\text {eff }}+a\left(\sqrt{N_{T}}-1\right)$.

Fig. 4 shows a colony in three different instants. We can see a change in the shape along the temporal evolution: from an undefined geometric form to a circular shape. Also, as described by Huergo et al. (2011, 2012), we can note that the characteristic cell size depends on its position in the aggregate. In addition, we notice that in large colonies, there exist cells piling up and forming multiple layers (figure not shown). In small colonies, at initial stages, the deviation of aggregates from the circular shape is accompanied by the formation of holes. These holes, in large colonies, are quickly filled, not directly influencing the growth dynamics, but the colony morphology.

In Fig. 5 we show a growth curve of three colonies of HCT-115. We collapsed the pre-pile-up mean radius of the colonies onto a single curve with the same parameters shown in the Fig. 2 . The dashed lines show the region of the growth curve where the pile up becomes evident. The stacking starts at time $t_{\mathrm{pile}}=287 \pm 9 \mathrm{~h}$ with an average radius $r_{\text {pile }}=380 \pm 30 \mu \mathrm{m}$.

Doing transformations in the data sets, by using Eqs. (2)-(4), we found a common sigmoidal behavior for the cell lineages shown in Fig. 6. Table 1 shows the parameters used to build this figure. ${ }^{5}$ We need to highlight the time window used: Brú et al. (2003) worked with a time window of about $1400 \mathrm{~h}$, while here we worked with about $330 \mathrm{~h}$. To make the data sets with about the same size, we did a cut off in the HT-29 data at about $\tau=7$. Nevertheless, the sigmoidal behavior is also compatible with Eq. (4) for reduced times greater than $\tau=7$. To see with more details, we refer the reader to the work of Costa et al. (2013), where the authors showed the non-reduced sigmoidal growth rate up to $t=1400 \mathrm{~h}(\tau \approx 19)$ for the HT-29 cell line.

\footnotetext{
${ }^{5}$ In practice, one obtains the derivative, by averaging the slope of adjacent points to each experimental data $\left(t_{i}, r_{i}\right)$, i.e., $\omega\left(t_{i}\right)=2^{-1}\left(\Delta r_{i+1} \Delta t_{i+1}^{-1}+\Delta r_{i} \Delta t_{i}^{-1}\right)$. At the extremes, $i=1$ and $i=i_{\max }$, we use only one term, and so we replace the factor 2 by 1.
} 

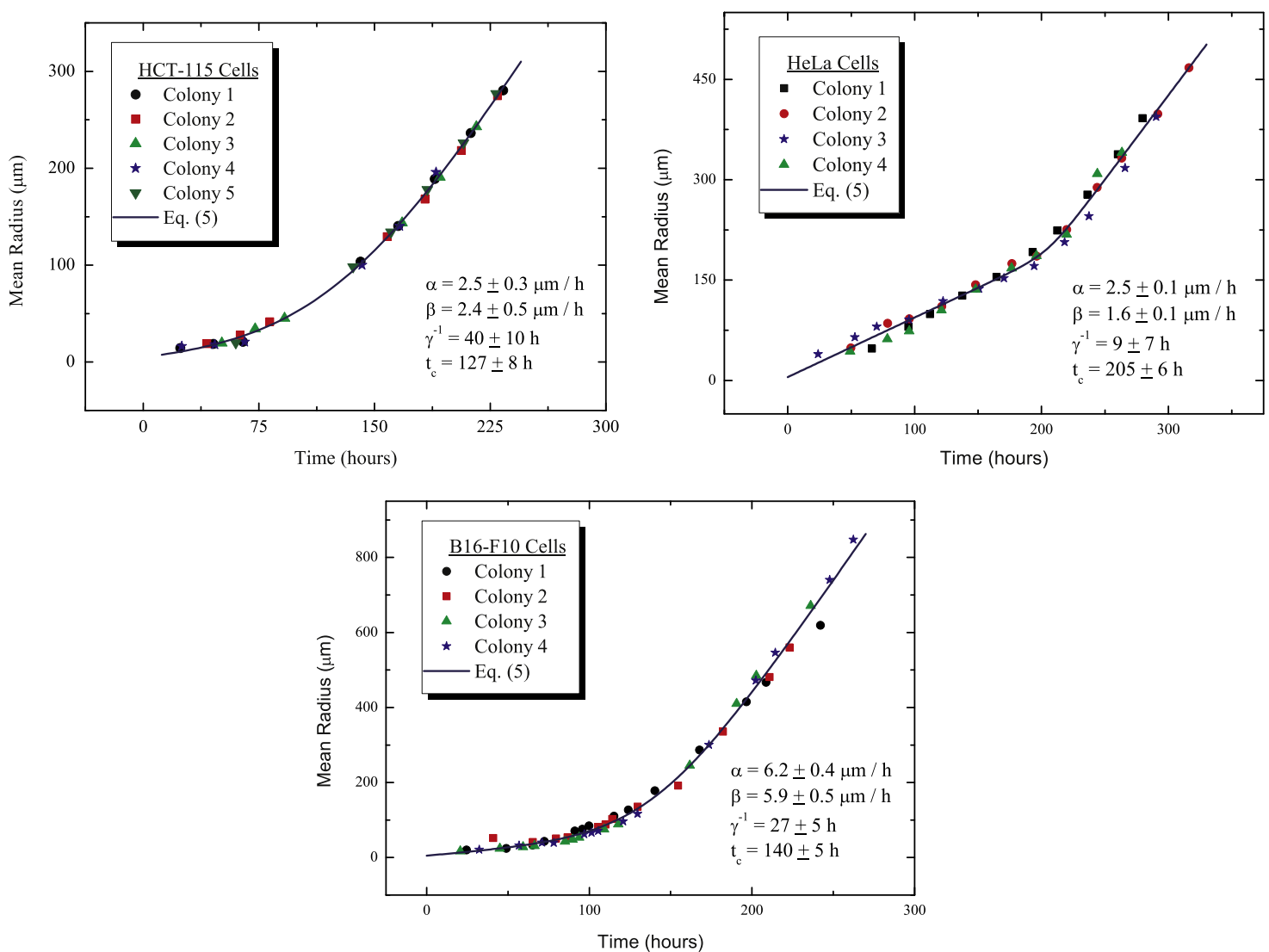

Fig. 2. Temporal evolution of the mean radius of cell aggregates. The upper left part shows the temporal evolution for HCT-115 cells, the upper right for HeLa lineage and the bottom for colonies of B16-F10. The colored symbols represent the experimental data points and the solid dark blue line is the fitting using the function given by Eq. (5). (For interpretation of the references to color in this figure caption, the reader is referred to the web version of this paper.)

Table 1

The parameters used in Fig. 6.

\begin{tabular}{lllll}
\hline Lineage & $\alpha$ & $\Gamma$ & $\gamma^{-1}$ & $t_{c}$ \\
\hline HT-29 & $1.96(1)$ & 0.633 & $60(10)$ & $459(8)$ \\
HeLa & $2.5(1)$ & 0.64 & $9(7)$ & $205(6)$ \\
HCT-115 & $2.5(3)$ & 0.96 & $40(10)$ & $127(8)$ \\
NIH-HN-13 & $3.6(1)$ & 0.70 & $16(2)$ & $140(*)$ \\
B16-F10 & $6.2(4)$ & 0.95 & $27(5)$ & $140(5)$ \\
\hline
\end{tabular}

The unit for $\alpha$ is $\mu \mathrm{m} / \mathrm{h}$; for $\gamma^{-1}$ and $t_{c}$ is $\mathrm{h} ; \Gamma$ is dimensionless.

$(\cdot)$ denotes the statistical error in the last significant digit; $(*)$ value was held fixed.
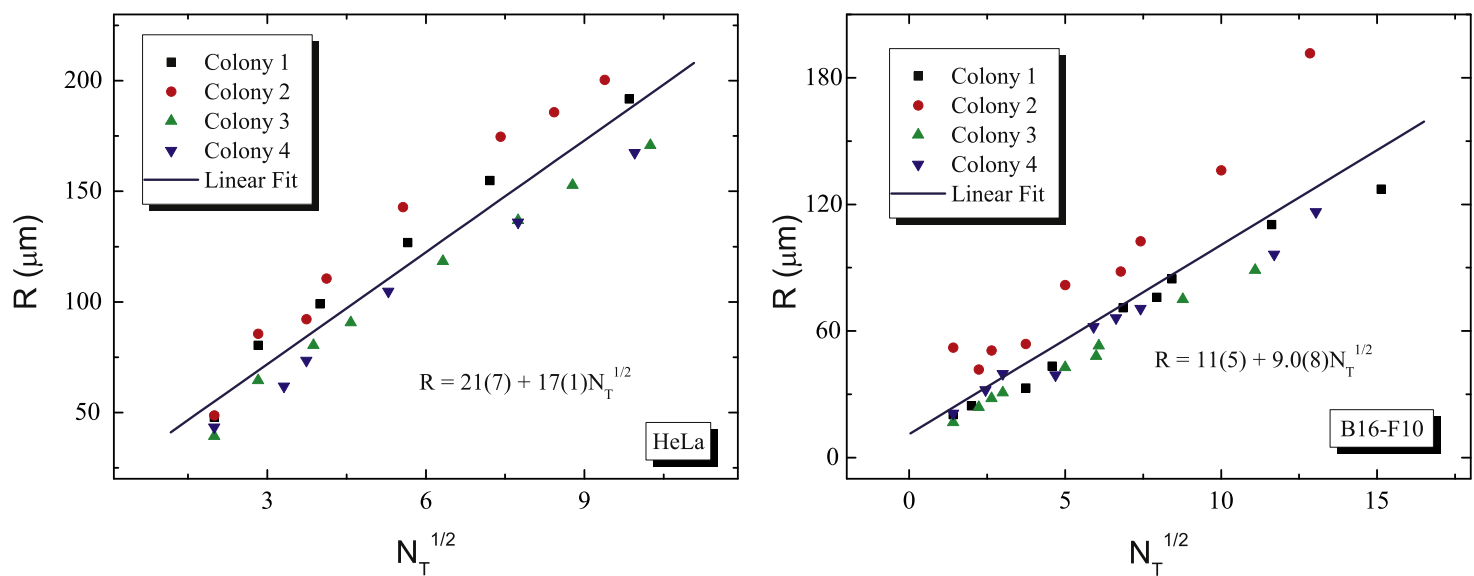

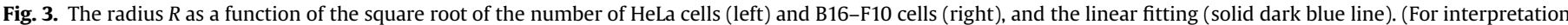
of the references to color in this figure caption, the reader is referred to the web version of this paper.) 

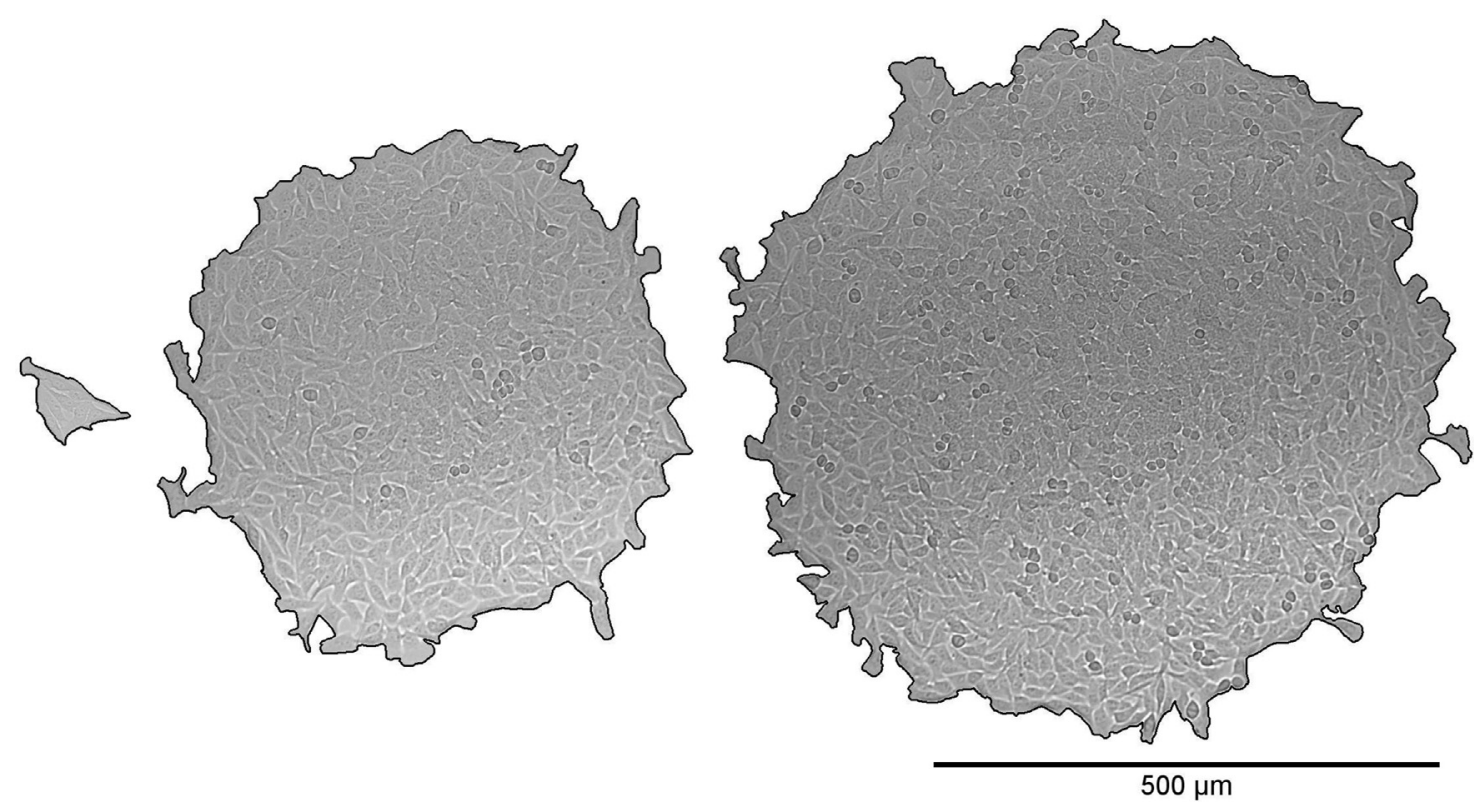

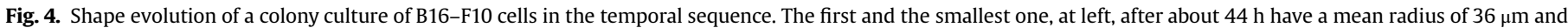

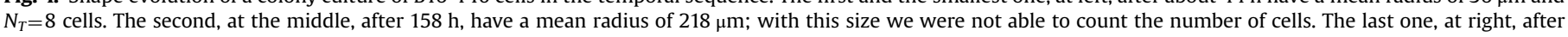
$166 \mathrm{~h}$ have a mean radius of $258 \mu \mathrm{m}$.

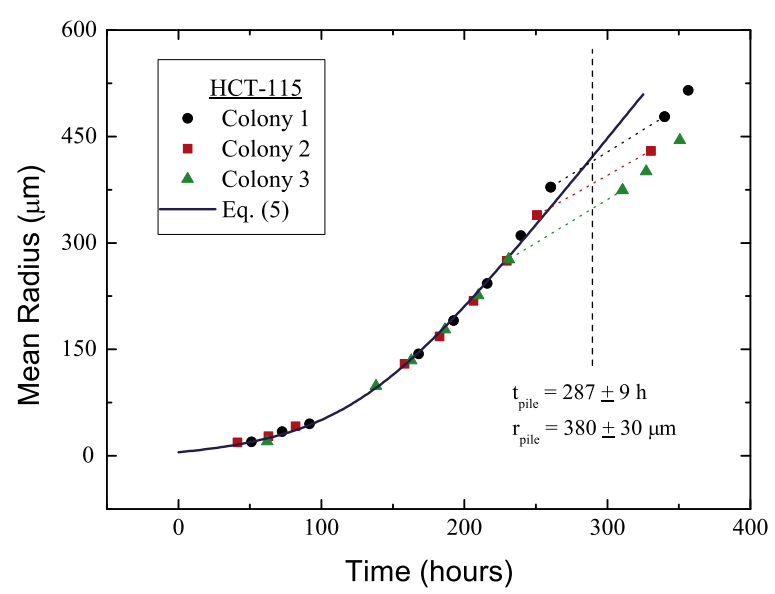

Fig. 5. Average radius for three colonies of HCT-115 cells fitted using the same parameters as in Fig. 2.

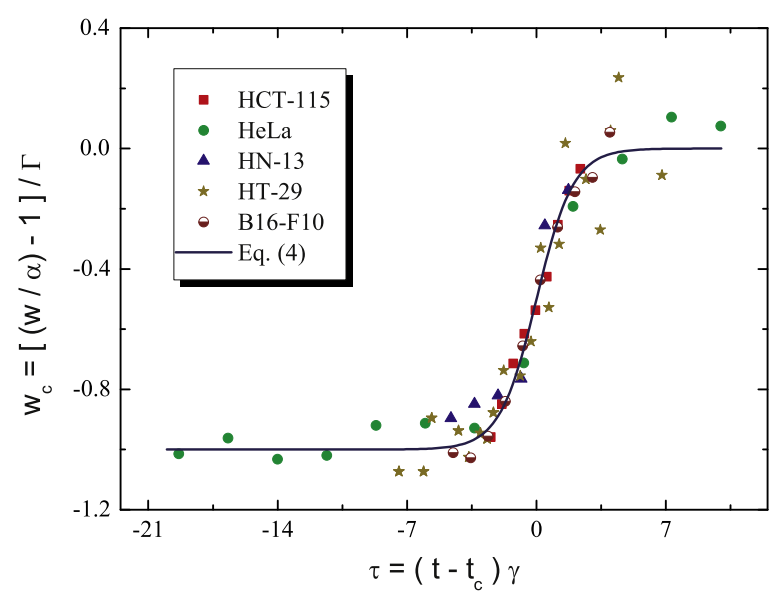

Fig. 6. The data collapse using reduced variables (rate and time) onto a single curve (solid dark blue line) for five cell types. The parameters used are summarized in Table 1. (For interpretation of the references to color in this figure caption, the reader is referred to the web version of this paper.)

\section{Discussion}

The data displacement on the time axis to construct the growth curves shown in Fig. 2 is a simplified procedure to correct effects due to colonies heterogeneity, such as cellular desynchronization, different adhesion times and initial cellular contact area. The values for $\alpha$ that we found to B16-F10 cells agree with the value found by Brú et al. (2003): $\alpha=5.83 \pm 0.01 \mu \mathrm{m} / \mathrm{h}$. However for the HeLa lineage, our results are about twice that obtained by Brú et al. (2003): $\alpha=1.34 \pm 0.01 \mu \mathrm{m} / \mathrm{h}$. One possible explanation for the difference in the growth rate for the Hela cells is in the culture medium: we used DMEM medium while Brú et al. used RPMI-1640 medium. A change in the culture medium may change some cellular properties (Tziampazis and Sambanis, 1994), including growth rates in established (Ostgaard et al., 1981) and primary cultures (Hagmann et al., 2013; Li et al., 2004).

We can find in the literature, a wide range of works using the RPMI-1640 and DMEM media in well established lines such as HeLa lineage. Therefore, to explain better the different growth rates between those reported by Brú et al. (2003) and Huergo et al. (2012), and in our work, we should do further investigation into the role of the medium in the regulation of growth rates. Following the same reasoning, other factors such as the change in the concentrations of serum (Durand, 1980), $\mathrm{CO}_{2}$, and glucose (Freyer and Sutherland, 1986; Jiang et al., 2005) have been reported as modifiers of the growth rate in spheroids, influenced mainly by the concentration of growth factors (Goustin et al., 1986). This must be valid also for monolayer cultures.

Our result for the growth rate of HeLa cells also is in disagreement with Huergo et al. (2012) result: $5 \pm 1 \mu \mathrm{m} / \mathrm{h}$. In this case, the difference between the experimental procedures is, besides the different culture media, the passage number. While we used low passages (between 2 and 20), Huergo et al. used a passage number of 44 . Campbell et al. (2002), showed that the number of passages is directly related with decreasing expression of estrogen receptor alpha (ER $\alpha$ ) in GH3/B6/F10 cells (rat pituitary tumor) - a difference of only 4 passages could significantly reduce the expression of $\operatorname{Er} \alpha$; this receptor has been correlated with cellular proliferation (Razandi et al., 1999). Whereas ER $\alpha$ is not expressed in HeLa cells (Fluoriot et al., 2000), the results shown above suggest that the number of passages plays an important role in regulating the growth rate. Generally, low passages are recommended in the study of cells (Masters, 
2000). More studies are necessary to examine these facts and we will do this in future works.

The experimental data shown in Fig. 2 fit well with the function given by Eq. (5), showing a good agreement with our rate modeling. An inspection of Eq. (1) and its solution Eq. (5) reveals three stages of colony growth. The first region is the stage in which the colony grows linearly with time, since $\omega(t) \approx \omega_{0}$. The second one, near $t_{c}$, is a crossover region, after that, the third one is where the radius converges to the linear asymptotic regime $r \propto \alpha t$. We believe that one could relate this regime change to a morphological transition, as observed in MDCK (Mardin-Darby canine kidney) cell lineage (Puliafito et al., 2012), and it could occur in all adherent cell lines. The condition of growth in this model is trivial, namely, $\alpha \geq \beta$ implying that $\Gamma \leq 1 ; \quad \Gamma=1$ is possible because of the factor $f_{0}=$ $\left[1+\exp \left(-\gamma t_{c}\right)\right]^{-1}$ that multiplies $\beta$ in Eq. (1) at $t=0$, satisfying $\omega_{0}>0$, condition to start the growth, although usually $f_{0} \rightarrow 1$. At the second region, one could replace the parameter $f_{0} \beta$ by an effective parameter $\beta_{\text {eff }}$, which we define as $\beta_{\text {eff }}=f \beta$, with $f=\left[1+\exp \left[\gamma\left(t-t_{c}\right]\right)\right]^{-1}$. We may relate this parameter with irregularities, such as protrusions and valleys, at the growth front, and some voids in the bulk, resulting in a non-circular colony. In some sense, one could relate $\beta_{\text {eff }}$ with the measurement of the aspect ratio such as studied by Huergo et al. (2011). Note that $\beta_{\text {eff }} \rightarrow f_{0} \beta$ when $t \ll t_{c}$, and $\beta_{\text {eff }} \rightarrow 0$ when $t \gg t_{c}$, so, for large $t$, the aggregate shape becomes close to circular. Notice that the sigmoidal rate (Eq. (1)) does not lead to the saturation of the growth curve, in agreement with the literature (Brú et al., 1998, 2003; Huergo et al., 2011, 2012; Drasdo and Hoëhme, 2005; Byrne and Drasdo, 2009; Drasdo et al., 2007; Radszuweit et al., 2009). The saturation is not a mechanism of the aggregate itself, but it is due to the boundary conditions of the culture flask. This fact contrasts with a Gompertzian growth profile (Laird, 1964; Winsor, 1932) in which saturation is expected, therefore our modeling does not apply to that type of tumor growth.

The parameter $R_{\text {eff }}$, defined with the function shown in Fig. 3, is different from $r_{0}$ (in Eq. (5)), the radius of a single cell at the time of adhesion to the culture plate. The observed cell spreading in contact with a surface explains the difference between these lengths (Odenthal et al., 2013; Cuvelier et al., 2007). As we can see in Fig. 3, the $R_{\text {eff }}$ of HeLa cells is approximately twice the value of B16-F10 cells, and one may relate this to the fact that HeLa cells can spread more than B16-F10 cells.

As we can see in Fig. 4, the inner cells in a large cluster have different sizes. Huergo et al. (2011, 2012) studied this in detail, and Puliafito et al. (2012) related it with the morphological transition. In this process, the compression exerted by the outer cells deforms the inner cells, causing area reduction, increasing the local density, and arresting the cell cycle. When the inner cells suffer sufficient stress, the pile up begins, caused both by the mechanism of compression and the ability of cells to escape from anoikis. In the B16-F10 cells, coordinated molecular mechanisms protect cells from anoikis (Goundiam et al., 2008, 2010).

As described in the theory of Galle et al. (2005), the behavioral change in the asymptotic linear region of the growth, as depicted in Fig. 5 for HCT-115 cells, suggests the formation of multiple layers. This change in behavior may be due to low adhesive energy between cells and plate, the compression exerted in the cells, and the ability of this cell line to escape from anoikis. Studies also suggest biochemical pathways and genetic mutations, as found in several types of tumors of the colon (HCT-115 is a lineage of colorectal cells). Which may lead to such resistance, and consequently, the propensity for the formation of multiple layers (Yan et al., 1997; Placzek et al., 2010). A contrasting fact to point out is that the growth velocity reduction in the linear region is not evident for B16-F10 and other cell lines: HeLa (Huergo et al., 2012), Vero (Huergo et al., 2011) and SW-480 (Galle et al., 2009); although the mechanisms required for the formation of multiple layers are the same as in HCT-115 cells. Possibly, this is due to a low cell to cell cohesive energy (Galle et al., 2009). It will require further studies to understand these mechanisms completely.

As we can see through of Fig. 6, fluctuations in the reduced growth rate extracted from HT-29 cell lineage, cultivated by Brú et al. (2003), are bigger than in other cell lines. These differences may be due to the number of colonies used to build the growth curve rate. While we used an average of five (HCT-115) or four (HeLa, B16-F10, NIH-HN-13) colonies, Brú et al. (2003) worked with just one colony.

Collapses into universal curves have been widely shown in cell culture properties, e.g., fractal behavior of aggregates (Brú et al., 1998, 2003; Huergo et al., 2010, 2011, 2012), ontogenic growth (Guiot et al., 2003, 2006), spreading of single cells (Odenthal et al., 2013; Cuvelier et al., 2007) and locomotion activity (Czirók et al., 1998). However, to our knowledge, this is the first time that one observes the collapse of growth rate functions, showing the same behavior to various cell lines. We believe that it happens to any adherent cell types growing in vitro; examples are MDCK cells (Puliafito et al., 2012) and HCT-116 (Galle et al., 2009), and may extend to other systems forming multilayer (Huergo et al., 2011, 2012; Galle et al., 2009) or spheroids (Drasdo and Hoëhme, 2005).

The sigmoidal common behavior may be caused by cooperative and competitive mechanisms, because of the characteristic shape of the rates, as those found in biochemical reactions. We believe that an essential mechanism is the formation of a critical nucleus, stabilized by cohesive forces favoring the adhesion to the plate (Galle et al., 2005, 2009; Cuvelier et al., 2007). Moreover, if the cohesive forces are weak, the cells easily become detached from (or move in) the aggregate, thus, adhesive forces are important to stabilize it; the total adhesive force increases dependent on the number of joined cells, thus, a cooperative effect may exist between these forces. After cells form the critical nucleus, the cohesive forces compress the inner cells, changing their average spreading. Therefore, we believe that the relation between cohesive and adhesive forces is the main cause for the morphological transition. One may relate cooperative mechanisms besides those of cohesive/adhesive forces, with other factors, such as the cooperation through the sharing of diffusible products (Axelrod et al., 2006). The competition, on the other hand, occurs mainly by the search for space in the colony border (Costa et al., 2013), since the inner cells became quiescents (Brú et al., 2003; Puliafito et al., 2012). This mechanism, in adherent colonies, is thought to be more important than other possible competition factors, such as nutrient competition (Brú et al., 2003). Therefore, one hypothesizes that the proliferating cells search for a larger number of contacts with locals that do not have other adherent cells. Costa et al. (2013) support this hypothesis with simulations on a lattice by adding a term in the probability of transition dependent on the number of local sites (neighboring of a dividing cell) without adherent cells.

Our effective sigmoidal rate approach implicitly considers possible death effects. However, it also supports models that do not explicitly consider cell death (Costa et al., 2013; Drasdo and Hoëhme, 2005; Block et al., 2007; Radszuweit et al., 2009), since the voids are filled quickly (Anon et al., 2012), without directly affecting the kinetics of the population (Rolli et al., 2012). Notice that through the relationship between the mean radius and the number of cells shown in Fig. 3, we can find $d R(t) / d t \propto N_{T}^{-1 / 2} d N_{T} / d t$ (Brú et al., 1998). With this relation, using the first part of Eq. (1) we obtain

$\frac{d}{d t} N_{T} \propto \omega(t) \sqrt{N_{T}}$,

which may represent a chemical reaction $Q \rightarrow T$ with rate $\omega(t)$; where $Q$ represents a local (available space) that may be filled by a daughter cell, and $T$ is a tumor cell. This process is analogous to fill an empty space represented by a vacant site in a lattice. As $Q$ is near the edges of the colony, we have the relation $[Q] \propto N_{T}^{1 / 2}$, which 
eventually results in Eq. (6). This description of the growth rate could be related with the mass rate of single cells (Mir et al., 2011; Son et al., 2012). However, more studies on these subjects are needed, and we are working to describe them from first principles.

\section{Conclusions and perspectives}

In this work, we modeled monolayer tumor growth in vitro with a sigmoid-type rate. By fitting the experimental data of the mean radius, we obtained the four parameters of the sigmoidal function. Through the collapse onto a single curve Eq. (4), we showed that the sigmoidal growth rate represents well the dynamics of several cell lineages. We did not take into account effects of diffusion and proliferation explicitly, as made by Simpson et al. (2013) and Treolar et al. (2014), because of the intrinsic difficulty of determining univocally the parameters (Maini et al. 2004a,b). Therefore, we assume an effective rate represented by a continuous function, also avoiding step-like functions, as for example, in Radszuweit et al. (2009). Our proposed growth rate has the following parameters: $\omega_{0}, \alpha, t_{c}$ and $\gamma$, and they are, respectively, the initial and final rates of growth, the instant at which the rate is equal to $\alpha-\beta / 2$ (related to a morphological transition, as observed by Puliafito et al., 2012), and the parameter $\gamma$ that tell us how fast the initial growth rate tends to $\alpha$. We report the parameters used for the adjustments in Table 1, however, we must stress that they are not absolute values for each lineage, since we displaced arbitrarily the growth curves shown in Fig. 2.

The collapse of the growth rates for the different cell lineages onto a single sigmoidal curve, shown in Fig. 6, suggests that Eq. (1) represent a universal behavior of adherent colonies growing in vitro. We believe that this behavior could be extended to other cells growing in monolayer (Puliafito et al., 2012; Galle et al., 2009) as well as to multilayer (Huergo et al., 2011, 2012; Galle et al., 2009), and, perhaps even in spheroids colonies (Drasdo and Hoëhme, 2005). Moreover, the sigmoidal shape, as discussed here, suggests that mechanisms such as cooperation and competition between cells within an aggregate are commonly present. Thus, we hope to have shown that the sigmoidal curve rate can store various information of interest for modeling in this research area. Our future studies will focus on obtaining the sigmoidal growth rate from first principles formulations and chemical kinetics related schemes.

\section{Acknowledgment}

F.H.S.C. is grateful to funding support from CAPES and we thank FAPESP(Grant no. 2012/03823-5) for financial assistance.

We are grateful to K. Lauterstein for careful reading the text, and to anonymous referees for the great improvement of their comments.

\section{Appendix A. Supplementary data}

Supplementary data associated with this paper can be found in the online version at http://dx.doi.org/10.1016/j.jtbi.2015.09.035.

\section{References}

Axelrod, R., Axelrod, D.E., Pienta, K.J., 2006. Evolution of cooperation among tumor cells. Proc. Natl. Acad. Sci. 103, 13474-13479.

Amar, M.B., Chatelain, C., Ciarletta, P., 2011. Contour instabilities in early tumor growth models. Phys. Rev. Lett. 106, 148101.

Anon, E., Serra-Picamal, X., Hersen, P., Gauthier, N.C., Sheetz, M.P., Trepat, X., Ladoux, B., 2012. Cell crawling mediates collective cell migration to close undamaged epithelial gaps. Proc. Natl. Acad. Sci. 109, 10891-10896.
Block, M., Schöll, E., Drasdo, D., 2007. Classifying the expansion kinetics and critical surface dynamics of growing cell populations. Phys. Rev. Lett. 99, 248101.

Brú, A., Albertos, S., Subiza, J.L., García-Asenjo, J.L., Brú, I., 2003. The universal dynamics of tumor growth. Biophys. J. 85, 2948-2961.

Brú, A., Pastor, J.M., Fernaud, I., Brú, I., Melle, S., Berenguer, C., 1998. Super-rough dynamics on tumor growth. Phys. Rev. Lett. 81, 4008-4011.

Byrne, H., Drasdo, D., 2009. Individual-based and continuum models of growing cell populations: a comparison. J. Math. Biol. 58, 657-687.

Byrne, H.M., 2010. Dissecting cancer through mathematics: from the cell to the animal model. Nat. Rev. Cancer 10, 221-230.

Campbell, C.H., Bulayeva, N., Brown, D.B., Gametchu, B., Watson, C.S., 2002. Regulation of the membrane estrogen receptor- $\alpha$ : role of cell density, serum, cell passage number, and estradiol. FASEB J. 116, 1917-1927.

Chatelain, C., Ciarletta, P., Amar, M.B., 2011. Morphological changes in early melanoma development: influence of nutrients, growth inhibitors and cell-adhesion mechanisms. J. Theor. Biol. 290, 46-59.

Costa, F.H.S., Campos, M., Aiéllo, O.E., da Silva, M.A.A., 2013. Basic ingredients for mathematical modeling of tumor growth in vitro: cooperative effects and search for space. J. Theor. Biol. 337, 24-29.

Cuvelier, D., Théry, M., Chu, Y.S., Dufour, S., Thiéry, J.P., Bornens, M., Nassoy, P., Mahadevan, L., 2007. The universal dynamics of cell spreading. Curr. Biol. 17, 694-699.

Czirók, A., Schelett, K., Madarász, E., Vicsek, T., 1998. Exponential distribution of locomotion activity in cell cultures. Phys. Rev. Lett. 81, 3038-3041.

Dawson, A., Hillen, T., 2006. Derivation of the tumour control probability (TCP) from a cell cycle model. Comput. Math. Methods Med. 7, 121-141.

Drasdo, D., Hoëhme, S., 2005. A single-cell-based model of tumor growth in vitro: monolayers and spheroids. Phys. Biol. 2, 133-147.

Drasdo, D., Hoehme, S., Bloch, M., 2007. On the role of physics in the growth and pattern formation of multi-cellular systems: what we can learn from individual-cell based models?. J. Stat. Phys. 128, 287-345.

Durand, R.E., 1980. Variable radiobiological response of spheroids. Radiat. Res. 81, 85-99.

Freyer, J.P., Sutherland, R.M., 1986. Regulation of growth saturation and development of necrosis in EMT6/Ro multicellular spheroids by the glucose and oxygen supply. Cancer Res. 46, 3504-3512.

Fluoriot, G., Brand, H., Denger, S., Metivier, R., Kos, M., Reid, G., Sonntag-Buck, V. Gannon, F., 2000. Identification of a new isoform of the human estrogen receptor-alpha (hER- $\alpha$ ) that is encoded by distinct transcripts and that is able to repress hER- $\alpha$ function 1. EMBO J. 19, 4688-4700.

Galle, J., Loeffler, M., Drasdo, D., 2005. Modeling the effect of deregulated proliferation and apoptosis on the growth dynamics of epithelial cell populations in vitro. Biophys. J. 88, 62-75.

Galle, J., Hoffmann, M., Aust, G., 2009. From single cells to tissue architecture-a bottom-up approach to modelling the spatio-temporal organisation of complex multi-cellular systems. J. Math. Biol. 58, 261-283.

Goundiam, O., Nagel, M.D., Vayssade, M., 2008. Study of cancer survival using na anti-adhesive substratum. Comput. Methods Biomech. Biomed. Eng. Suppl. 1, 105-106.

Goundiam, O., Nagel, M.D., Vayssade, M., 2010. Growth and survival signalling in B16F10 melanoma cells in 3D culture. Cell Biol. Int. 34, 385-391.

Goustin, A.S., Leof, E.B., Shipley, G.D., Moses, H.L., 1986. Growth factors and cancer. Cancer Res. 46, 1015-1029.

Guiot, C., Degiorgis, P.G., Delsanto, P.P., Gabriele, P., Deisboeck, T.S., 2003. Does tumor growth follow a Universal Law? J. Theor. Biol. 225, 147-151.

Guiot, C., Delsanto, P.P., Carpinteri, A., Pugno, N., Mansury, Y., Deisboeck, T.S., 2006. The dynamic evolution of the power exponent in a universal growth model of tumors. J. Theor. Biol. 240, 459-463.

Hagmann, S., Moradi, B., Frank, S., Dreher, T., Kämmerer, P.W., Richter, W., Gotterbarm, T., 2013. Different culture media affect growth characteristics, surface markers distribution and chondrogenic differentiation of human bone marrowderived mesenchymal stromal cells. BMC Musculoskelet. Disord. 14, 223.

Huergo, M.A.C., Pasquale, M.A., Bolzán, A.E., Arvia, A.J., González, P.H., 2010. Morphology and dynamic scaling of cell colonies with linear growth fronts. Phys. Rev. E 82, 031903.

Huergo, M.A.C., Pasquale, M.A., González, P.H., Bolzán, A.E., Arvia, A.J., 2011. Dynamics and morphology characteristics of cell colonies with radially spreading growth fronts. Phys. Rev. E 84, 021917.

Huergo, M.A.C., Pasquale, M.A., González, P.H., Bolzán, A.E., Arvia, A.J., 2012. Growth dynamics of cancer cells colonies and their comparison with noncancerous cells. Phys. Rev. E 85, 011918.

Jiang, Y., Pjesivac-Grbovic, J., Cantrell, C., Freyer, J.P., 2005. A multiscale model for avascular tumor growth. Biophys. J. 89, 3884-3894.

Kim, M., Reed, D., Rejniak, K.A., 2014. The formation of tight tumor clusters affects the efficacy of cell cycle inhibitors: a hybrid model study. J. Theor. Biol. 352, 31-50.

Laird, A.K., 1964. Dynamics of tumor growth. Br. J. Cancer 18, 490-502.

Leith, J.T., Quaranto, L., Padfield, G., Michelson, S., Hercbergs, A., 1993. Radiobiological studies of PC-3 and DU-145 human prostate cancer cells: X-ray sensitivity in vitro and hypoxic fractions of xenografted tumor in vivo. Int. J. Radiat. Oncol. 25, 283-287.

Li, N.F., Wilbanks, G., Balkwill, F., Jacobs, I.J., Dafou, D., Gayther, S.A., 2004. A modified medium that significantly improves the growth of human normal ovarian surface epithelial (OSE) cells in vitro. Lab. Investig. 84, 923-931.

Maini, P.K., McElwain, D.L.S., Leavesley, D.I., 2004a. Traveling wave model to interpret a wound-healing cell migration assay for human peritoneal mesothelial cells. Tissue Eng. 10, 475-482. 
Maini, P.K., McElwain, D.L.S., Leavesley, D.I., 2004b. Traveling waves in a wound healing assay. Appl. Math. Lett. 17, 575-580.

Masters, J.R.W., 2000. Animal Cell Culture: A Practical Approach. Oxford University Press, Oxford.

Mir, M., Wang, Z., Shen, Z., Bednardz, M., Bashir, R., Golding, I., Prasanth, S.G., Popescu, G., 2011. Optical measurement of cycle-dependent cell growth. Proc. Natl. Acad. Sci. 108, 13124-13129.

Odenthal, T., Smeets, B., Liedekerke, P.V., Tijskens, E., Oosterwyck, H.V., Ramon, H., 2013. Analysis of initial cell spreading using mechanistic contact formulations for a deformable cell model. PLOS Comput. Biol. 9, e1003267.

Ostgaard, K., Wibe, E., Lindmo, T., Eik-Nes, K.B., 1981. Effects of steroids and different culture media on cell cycle of the androgen-sensitive human cell line NHIK3025. J. Cell Sci. 48, 281-290.

Placzek, W.J., Wein, J., Kitada, S., Zhai, D., Reed, J.C., Pellecchia, M., 2010. A survey of the anti-apoptotic Blc-2 subfamily expression in cancer types provides a platform to predict the efficacy of Bcl-2 antagonists in cancer therapy. Cell Death Dis. 1, e40.

Poplawski, N.J., Agero, U., Gens, J.S., Swat, M., Glazier, J.A., Anderson, A.R.A., 2009. Front instabilities and invasiveness of simulated avascular tumors. Bull. Math. Biol. 71, 1189-1227.

Puliafito, A., Hufnagel, L., Neveu, P., Streichan, S., Sigal, A., Fygenson, D.K., Shraiman, B.I., 2012. Collective and single cell behavior in epithelial contact inhibition. Proc. Natl. Acad. Sci. 109, 739-744.

Radszuweit, M., Block, M., Hengstler, J.G., Schöll, E., Drasdo, D., 2009. Comparing the growth kinetics of cell populations in two and three dimensions. Phys. Rev. E 79, 051907.
Razandi, M., Pedram, A., Greene, G.L., Levin, E.R., 1999. Cell membrane and nuclear estrogen receptors (ERs) originate from a single transcript: studies of ER $\alpha$ and ER $\beta$ expressed in Chinese hamster ovary cells. Mol. Endocrinol. 13, 307-319.

Rolli, C.G., Nakayama, H., Yamaguchi, K., Spatz, J.P., Kemkemer, R., Nakanishi, J. 2012. Switchable adhesive substrates: revealing geometry dependence in collective cell behavior. Biomaterials 33, 2409-2418.

Simpson, M.J., Treloar, K.K., Binder, B.J., Haridas, P., Manton, K.J., Leavesley, D.I., McElwain, D.L.S., Baker, R.E., 2013. Quantifying the roles of cell motility and cell proliferation in a circular barrier assay. J. R. Soc. Interface 10, 20130007.

Son, S., Tzur, A., Weng, Y., Jorgensen, P., Kim, J., Kirschner, M.W., Manalis, S.R., 2012. Direct observation of mammalian cell growth and size regulation. Nat. Methods 9, 910-913.

Tziampazis, E., Sambanis, A., 1994. Modeling of cell culture processes. Cytotechnology 14, 191-204.

Treolar, K.K., Simpson, M.J., McElwain, D.L.S., Baker, R.E., 2014. Are in vitro estimates of cell diffusivity and cell proliferation rate sensitive to assay geometry? J. Theor. Biol. 356, 71-84.

Unkelbach, J., Menze, B.H., Konukoglu, E., Dittmann, F., Ayache, N., Shih, H.A., 2014 Radiotherapy planning for glioblastoma based on a tumor growth model: implications for spatial dose redistribution. Phys. Med. Biol. 59, 771-789.

Winsor, C.P., 1932. The Gompertz curve as a growth curve. Proc. Natl. Acad. Sci. 18 (1), $1-8$.

Yan, Z., Chen, M.X., Perucho, M., Friedman, E., 1997. Oncogenic Ki-ras but not oncogenic Ha-ras blocks integrin $\beta 1$-chain maturation in colon epithelial cells. J. Biol. Chem. 272, 30928-30936. 